\title{
The effect of electroacupuncture on hemodialysis patients with restless legs syndrome (RLS): study protocol for a randomized crossover trial
}

Jia-Ming Chen ( $D$ 149353@gmail.com )

China Medical University https://orcid.org/0000-0003-2920-6697

Ping-Fang Chiu

Changhua Christian Medical Foundation Changhua Christian Hospital

\section{Yu-Jun Chang}

Changhua Christian Medical Foundation Changhua Christian Hospital

\section{Po-Chi Hsu}

China Medical University

Chia-Chu Chang

Kuang Tien General Hospital

Lun-Chien Lo

China Medical University

\section{Study protocol}

Keywords: Acupuncture, Restless leg syndrome, Hemodialysis, Heart rate variability

Posted Date: October 1st, 2020

DOl: https://doi.org/10.21203/rs.3.rs-32357/v1

License: (9) (i) This work is licensed under a Creative Commons Attribution 4.0 International License.

Read Full License 


\section{Title}

The effect of electroacupuncture on hemodialysis patients with restless legs syndrome $(R L S)$ : study protocol for a randomized crossover trial

Jia-Ming Chen ${ }^{12}$, Ping-Fang Chiu ${ }^{3}$, Yu-Jun Chang ${ }^{4}$, Po-Chi Hsu ${ }^{6}$, Chia-Chu Chang ${ }^{5}$, LunChien $\mathrm{LO}^{67}$

${ }^{1}$ Graduate Institute of Chinese Medicine, China Medical University, Taichung, Taiwan.

${ }^{2}$ Department of Traditional Chinese Medicine, Changhua Christian Hospital, Changhua, Taiwan.

${ }^{3}$ Nephrology Division, Department of Internal Medicine, Changhua Christian Hospital, Changhua, Taiwan.

${ }^{4}$ Epidemiology and Biostatistics Center, Changhua Christian Hospital, Changhua, Taiwan

${ }^{5}$ Division of Nephrology, Department of Internal Medicine, Kuang Tien General Hospital, Taichung, Taiwan.

${ }^{6}$ School of Chinese Medicine, China Medical University, Taichung, Taiwan.

${ }^{7}$ Department of Chinese Medicine, China Medical University Hospital, Taichung, Taiwan.

\section{Abstract}

- Background: The aim of the present study was to evaluate the effects of electroacupuncture (EA) in hemodialysis patients with restless leg syndrome (RLS) by heart rate variability (HRV) monitor.

- Methods: 112 subjects who were hemodialysis patients with RLS will be divided into 2 groups: experimental and control. Each subject will receive the treatment relevant to their group 2 times a week for 4 weeks. After 4 weeks of treatment the subject will enter a 2-week washout period, after which the subjects will switch groups. Measurements will include HRV recordings, International Restless Legs Syndrome Rating Scale (IRLSRS) and Insomnia Severity Index (ISI).

- Discussion: This study is the first investigation to analyze the relationship between EA and the change of $\mathrm{HRV}$ by an objective monitor. If the findings of the current trial are positive, this study will also help support an effective, safe and cheap approach to clinical treatment of this challenging disorder, help foster improved understanding the relationship between RLS and HRV, and ultimately contribute to elucidate the mechanisms of EA.

- Trial registration: ClinicalTrials.gov Identifier: NCT04356794; registration date: April 22, 2020. 


\section{Keywords}

Acupuncture, Restless leg syndrome, Hemodialysis, Heart rate variability

\section{Administrative information}

\begin{tabular}{|l|l|}
\hline Title $\{1\}$ & $\begin{array}{l}\text { The effect of electroacupuncture on hemodialysis patients } \\
\text { with restless legs syndrome(RLS): study protocol for a } \\
\text { randomized crossover trial }\end{array}$ \\
\hline Trial registration \{2a and 2b\}. & $\begin{array}{l}\text { 1.Institutional review boards of the Changhua Christian } \\
\text { Hospital (No. : 170217). } \\
\text { 2.ClinicalTrials.gov Identifier: NCT04356794 }\end{array}$ \\
\hline Protocol version $\{3\}$ & $\begin{array}{l}\text { Protocol No. : Y_105_0300 / CCH IRB No. : 170217 } \\
\text { Date of Approval: Aug 23, 2019 } \\
\text { Informed consent: V8 / Jul 26,2019 }\end{array}$ \\
\hline Funding $\{4\}$ & $\begin{array}{l}\text { This study was supported by grants from the Changhua } \\
\text { Christian Hospital, Taiwan (No. : 92419). }\end{array}$ \\
\hline $\begin{array}{l}\text { Author details }\{5 a\} \\
\text { information for the trial } \\
\text { sponsor }\{5 b\}\end{array}$ & $\begin{array}{l}\text { JMC contributed to conception, design of the study, and } \\
\text { manuscript writing. } \\
\text { PFC, YCC and LCL participated in the study conception } \\
\text { and the analysis of the data. All authors read and } \\
\text { approved the final manuscript in the Authors' } \\
\text { contributions section. }\end{array}$ \\
\hline $\begin{array}{l}\text { This study was supported by grants from the Changhua } \\
\text { Christian Hospital, Taiwan }\end{array}$ \\
\hline
\end{tabular}




\begin{tabular}{|l|l|}
\hline Role of sponsor $\{5 c\}$ & $\begin{array}{l}\text { The Changhua Christian Hospital will have ultimate } \\
\text { authority over any of these activities. } \\
\text { Address: } 135 \text { Nanxiao St., Changhua City, Changhua } \\
\text { County } 500, \text { Taiwan (R.O.C.) } \\
\text { Tel: } 886-4-723-8595\end{array}$ \\
\hline
\end{tabular}

\section{Introduction}

\section{Background and rationale $\{6 \mathrm{a}\}$}

Restless legs syndrome (RLS), also known as Willis-Ekbom disease is a neurological sensory disorder with symptoms involving an often unpleasant or irresistible urge to move the legs that occurs during periods of inactivity, particularly in the evenings, and is transiently relieved by movement [1]. A large population-based study in Europe and the United States found that $37 \%$ of patients with RLS symptoms had moderate to severe disease [2].

RLS can be primary or can occur as a secondary disorder in association with medical conditions, including low iron levels, kidney failure, Parkinson's disease, diabetes mellitus, rheumatoid arthritis, and pregnancy [3]. Studies in patients with dialysis showed a prevalence of RLS ranging from 12 to $62 \%$, which is significantly higher compared to the general population [4-8]. A previous study concluded that the high prevalence of RLS among dialysis patients and the associations between the severity of RLS and the risk of new cardiovascular events and higher short-term mortality [9].

Heart rate is modulated through effects of sympathetic and parasympathetic nervous systems, and analysis of changes in heart rate over time provides information about autonomic function [10]. An important non-invasive method used widely to study these changes is heart rate variability (HRV). A previous study demonstrated that heart rate variability is related to RLS [11]. A recent study indicated that a decrease in HRV is typically found in dialysis patients with abnormal HRV being considered an independent risk factor for mortality [12].

Several previous studies have showed a relationship between acupuncture and heart rate variability in RLS. Yamamoto et al. reported that standard acupuncture might improve the abnormal leg activity in RLS patients [13]. Koo et al. reported that electroacupuncture 
(EA) significantly increased in the LF/HF ratio in healthy volunteers [14]. To our best knowledge, the HRV analysis on the therapeutic effect of EA in hemodialysis patients with RLS has not been performed earlier.

For these reasons, we will collaborate with a hemodialysis room in order to investigate HRV evaluation of EA treatment on RLS in hemodialysis patients. In this study, we decided to conduct a patient-assessor-blinded, randomized, sham controlled crossover pilot trial.

\section{Objectives $\{7\}$}

The aim of this study is to assess the effectiveness of EA in the treatment of RLS. We evaluated the effects of EA in hemodialysis patients with RLS by HRV recordings, IRLSRS and ISI. All adverse events will also be assessed.

\section{Trial design $\{8\}$}

This study is a randomized-controlled crossover trial with a 2-week washout period between each crossover phase.

\section{Methods: Participants, interventions and outcomes}

\section{Study setting $\{9\}$}

The trials will be conducted at the Changhua Christian Hospital. This study will adhere to the recommendations of the Consolidated Standards of Reporting Trials (CONSORT) [15] to allow for greater completeness, transparency and accuracy of reporting. The protocol for this study has been registered in the Clinical Trials register (ClinicalTrials.gov Identifier: NCT04356794). A flow diagram of the trial is shown in Fig. 1. 


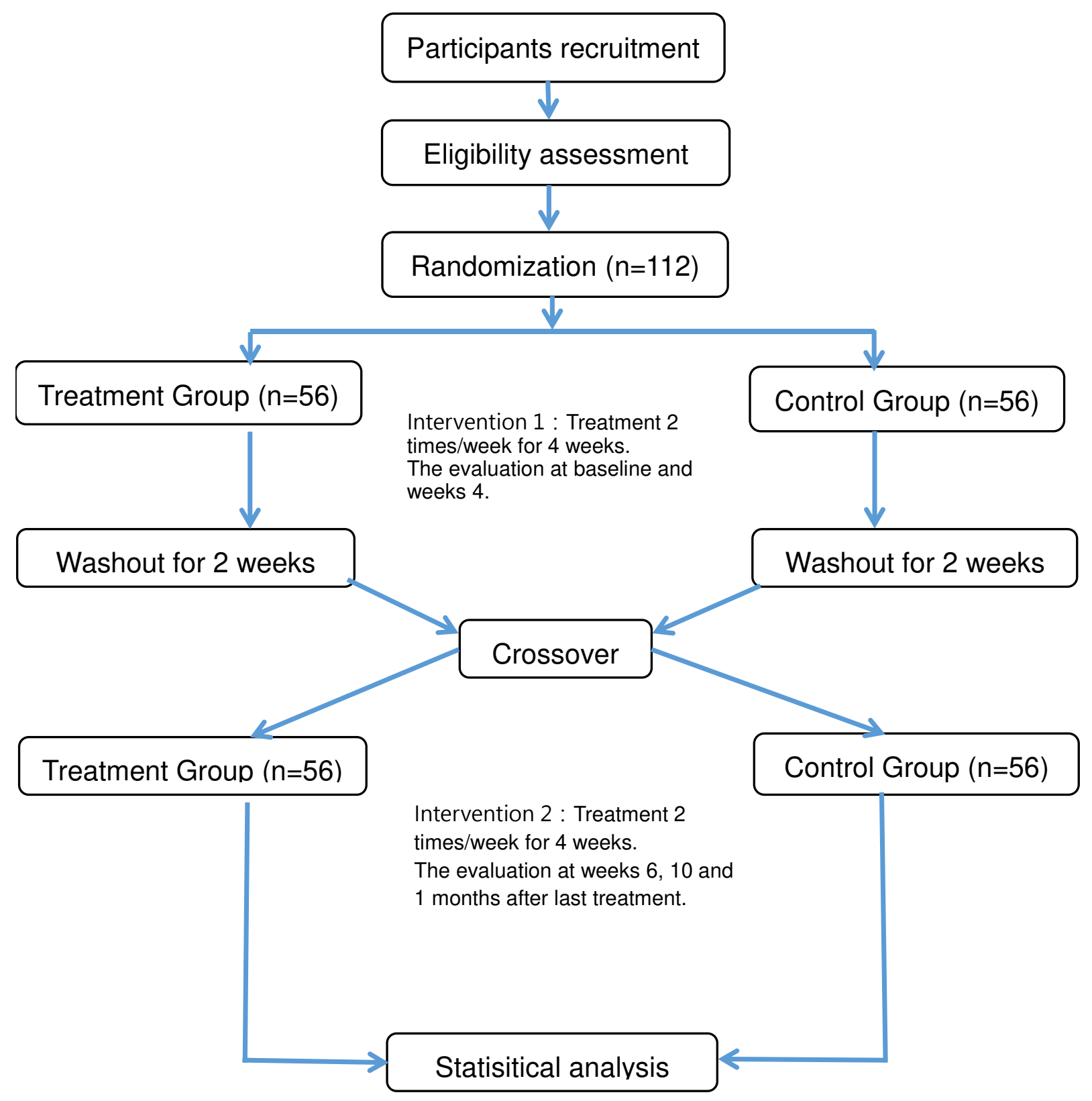

Fig. 1 Flowchart of the study design 


\section{Eligibility criteria $\{10\}$}

Subjects who voluntarily sign a consent form will undergo the trial according to the study design. When a subject is determined to be fit for participation based on inclusion and exclusion criteria, the subject will be randomly assigned to 1 of 2 groups in a ratio of 1:1. One group is the true EA experimental group and the other is the sham EA control group. Each group will receive their treatment 2 times a week for 4 weeks. After 4 weeks of treatment and a 2-week washout period, the control group will switch to the experimental group while the experimental group will switch to the control group. The subjects then undergo the treatment of their new group for another 4 weeks.

Treatment and assessment will be performed independently and the practitioners will not be involved in assessing the outcome of the treatment. The subjects, the outcome assessors and the statistician performing the data analyses will be blinded to the treatment allocation throughout the study.

\section{Ethics}

This research protocol adheres to the principles of the Declaration of Helsinki and has been approved by the institutional review boards of the Changhua Christian Hospital (No. : 170217). Informed consent will be obtained from each participant before any treatment is given. All subjects will have the right to withdraw from the study at any time.

\section{Inclusion criteria}

The inclusion criteria were as follows:

1. Informed consent to participate in the study;

2. Between the ages of $20-80$ years;

3. A mean frequency of RLS symptoms during the last 6 months of more than twice per week;

4. A score of at least 20 on the International Restless Legs Syndrome Rating Scale(corresponding to severe RLS,IRLSRS);

5. A history of chronic renal failure treated with regular and continuous hemodialysis for at least 3 months;

6. No acupuncture treatment in the last one month.

\section{Exclusion criteria}

The exclusion criteria were as follows: 
1. An unwillingness to continue to participate in the study;

2. Vascular access in the leg area, such as an arteriovenous shunt;

3. The presence of peripheral neuropathy and vascular problems in the lower extremities;

4. Currently taking drugs for RLS, such as dopamine agonists, benzodiazepines, opioids, and gabapentin;

5. A history of other motor disorders, such as Parkinson's disease, dyskinesia, and dystonia;

6. With an artificial cardiac pacemaker;

7. Pregnancy or breastfeeding.

\section{Randomization and allocation concealment}

After initial assessments, subjects will be assigned to 1 of 2 groups with a 1:1 allocation ratio according to a computer-generated randomization list. The group each subject is allocated to will be concealed in sequentially numbered sealed opaque envelopes. The envelopes will only be opened after the subject has completed baseline clinical assessments. Randomization allocation will be concealed from the physician, subjects and evaluators.

\section{Who will take informed consent? $\{26 \mathrm{a}\}$}

The research assistants and the principal investigator.

Additional consent provisions for collection and use of participant data and biological specimens $\{26 \mathrm{~b}\}$

Not applicable, no samples collected.

\section{Interventions}

\section{Explanation for the choice of comparators $\{6 \mathrm{~b}\}$}

1.The period on placebo does not entail any additional risk of serious or irreversible harm to the patient.

2.The patient is capable of giving, and gives, fully informed consent.

3.The patient may request conventional treatment at any stage, or may be placed on such treatment by the treating physician.

Intervention description $\{11 \mathrm{a}\}$ 


\section{Experimental group: electroacupuncture}

Subjects will receive $8 \mathrm{EA}$ treatments. Treatments will be performed 2 times a week for 4 weeks. Based on the theory of Traditional Chinese Medicine (TCM) and clinical literature on acupuncture therapy for the obese, the acupoints are as follows: ST36 (Zusanli), GB34 (Yanglingquan), SP9 (Yinlingquan), SP6 (Sanyinjiao), BL57 (Chengshan) and KI9 (Zhubin). All acupoints are localized according to the WHO Standard Acupuncture Locations and exhibited in Table 1 and Fig.2. Treatment will be performed by licensed acupuncturists who have at least five years of experience in acupuncture. The sterile disposable acupuncture needles (length of $40 \mathrm{~mm}$, diameter of $0.27 \mathrm{~mm}$ ) were developed by Tennyson Medical Instrument Developing Co., Ltd, Taiwan.

The acupuncture points were stimulated by needle insertion, then manipulations of twirling, lifting, and thrusting performed on all needles for at least $30 \mathrm{~s}$ to reach De qi (a compositional sensation including soreness, numbness, distention, and heaviness). Among the seven acupunctural points mentioned above, only ST36 (Zusanli), GB34 (Yanglingquan), SP9 (Yinlingquan) and KI9 (Zhubin) with electrical stimulation are conducted. The frequency of electrical stimulation is $2 \mathrm{~Hz}$, and the intensities of the stimulation are below $9.8 \mathrm{~mA}$ for $15 \mathrm{~min}[16,17]$. 
Table 1 Locations of acupoints in acupuncture group

\begin{tabular}{|l|l|}
\hline \multicolumn{1}{|c|}{ Acupoints } & \multicolumn{1}{c|}{ Locations } \\
\hline $\begin{array}{l}\text { Zusanli } \\
\text { (ST36) }\end{array}$ & $\begin{array}{l}3 \text { cun }^{\text {a }} \text { directly below Dubi (ST35), and one finger-breadth lateral } \\
\text { to the anterior border of the tibia }\end{array}$ \\
\hline $\begin{array}{l}\text { Yanglingquan } \\
\text { (GB34) }\end{array}$ & $\begin{array}{l}\text { On the lateral aspect of the lower leg, in the depression anterior } \\
\text { and inferior to the head of the fibula. }\end{array}$ \\
\hline $\begin{array}{l}\text { Yinlingquan } \\
\text { (SP9) }\end{array}$ & $\begin{array}{l}\text { On the medial aspect of the lower leg, in the depression of the } \\
\text { lower border of the medial condyle of the tibia. }\end{array}$ \\
\hline $\begin{array}{l}\text { Sanyinjiao } \\
\text { (SP6) }\end{array}$ & $\begin{array}{l}\text { On the medial aspect of the lower leg, 3 cun above the medial } \\
\text { malleolus, on the posterior border of the medial aspect of the } \\
\text { tibia. }\end{array}$ \\
\hline $\begin{array}{l}\text { Chengshan } \\
\text { (BL57) }\end{array}$ & $\begin{array}{l}\text { On the posterior midline of the lower leg between UB 40 and UB } \\
\text { 60. On the half way between the crease at the back of the knee } \\
\text { and the ankle. }\end{array}$ \\
\hline $\begin{array}{l}\text { Zhubin } \\
\text { (KI 9) }\end{array}$ & $\begin{array}{l}\text { On the medial aspect of the lower leg, 5 cun directly above KID 3, } \\
\text { on the line connecting KID 3 and KID 10, at the medial and } \\
\text { inferior end of the belly of m. gastrocnemius. }\end{array}$ \\
\hline a cun $\approx 20 \mathrm{~mm})$ \\
is defined as the width of the interphalangeal joint of patient's thumb
\end{tabular}

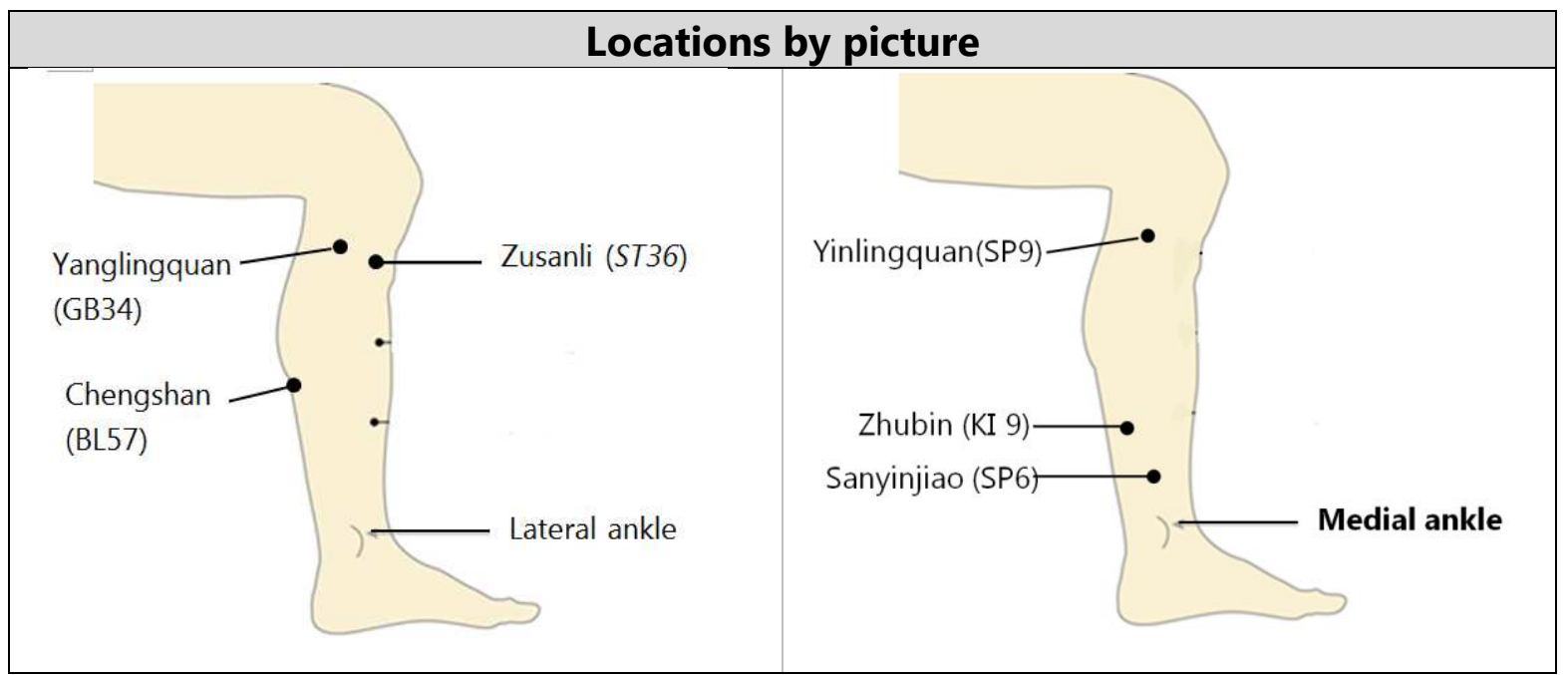

Fig. 2 Locations of acupoints 


\section{Control group: sham electroacupuncture}

Although it remains methodologically challenging to establish an inert and concealable sham EA control, superficial insertion at non-acupuncture points plus no electrical stimulation is the most commonly used approach for administering sham treatments among EA trials on the basis of a literature review[18, 19]. Sham EA in this study is delivered by on non-acupuncture points plus superficial insertion plus no electrical stimulation.

All interventions shall be the same as those of the experimental group except the location of the acupoints. The sham acupoints shall be about $1.0 \mathrm{~cm}$ from the real acupoints in the experimental group and are spots that do not belong to standard acupoints on the body and are not suitable for actual acupuncture based on anatomical and other conditions, without needle manipulation for De qi. Besides, the dummy mode of the apparatus was used, in which it produced sound signals but no electrical current. All application will be performed by the same physician.

\section{Criteria for discontinuing or modifying allocated interventions $\{11 \mathrm{~b}\}$}

1. Electroacupuncture will be discontinued if the patients suffer from any adverse events (AEs).

2. The number of complications reported to the FDA is relatively low, given that millions of people receive acupuncture treatment each year. You have the right to withdraw from this study at any time during the research, which will not affect your rights and you will not be discriminated or retaliated against.

\section{Strategies to improve adherence to interventions $\{11 \mathrm{c}\}$}

Through regular assessment of patient adherence by itself can lead to increased patient adherence.

\section{Relevant concomitant care permitted or prohibited during the trial $\{11 \mathrm{~d}\}$}

Drugs prescribed for dialysis patients are permitted except drug treatments for RLS during the trial.

\section{Provisions for post-trial care $\{30\}$}

The research assistant will keep tracking the direction (conditions) of post-treatment 
participants. Any personal information will remain confidential in a safe.

\section{Outcomes $\{12\}$}

The primary outcome was the change in activity level of HRV in the RLS patients. The second outcome was the change in IRLSRS and ISI scores.

This study monitored physiological parameter changes after EA treatment. Blood pressures, heart rate $(\mathrm{HR})$ and $\mathrm{HRV}$ were measured using an ANSWatch ${ }^{\circledR}$ monitor developed by Taiwan Scientific Corp./ Ledron Technology Corp., Taiwan. The HRV is analyzed in both time and frequency domains to give rise to parameters that are linked to total ANS activity [HRV or SDNN (standard deviation of NN intervals); TP (Total Power)], sympathetic activity (LF \%), parasympathetic (or vagal) activity (HF \%), and sympathyvagal balance (LF/HF) indexes.

The IRLS Rating Scale was developed by the International Restless Legs Syndrome Study Group (IRLSSG) to assess the severity of a patient's RLS symptoms. 10 questions are listed in the questionnaire and scored from 4 (usually 'very severe') to 0 (usually none). The sum of the item scores is graded from 0 to 40 . The scoring criteria are: Mild (score 110); Moderate (score 11-20); Severe (score 21-30); Very severe (score 31-40)[20].

The other questionnaire is ISI designed as a brief screening tool for insomnia. The seven-item questionnaire asks respondents to rate the nature and symptoms of their sleep problems using a Likert-type scale. The sum of the item scores is graded from 0 (no clinically significant insomnia) to 28(severe)[21].

\section{Follow-up}

Follow-up observations will be conducted before the trials, during the treatment, and 1 month after the last treatment. The schedule of enrolment, intervention, and assessments is shown in table 2 . 
Table 2 Schedule of enrollment, interventions, and assessments

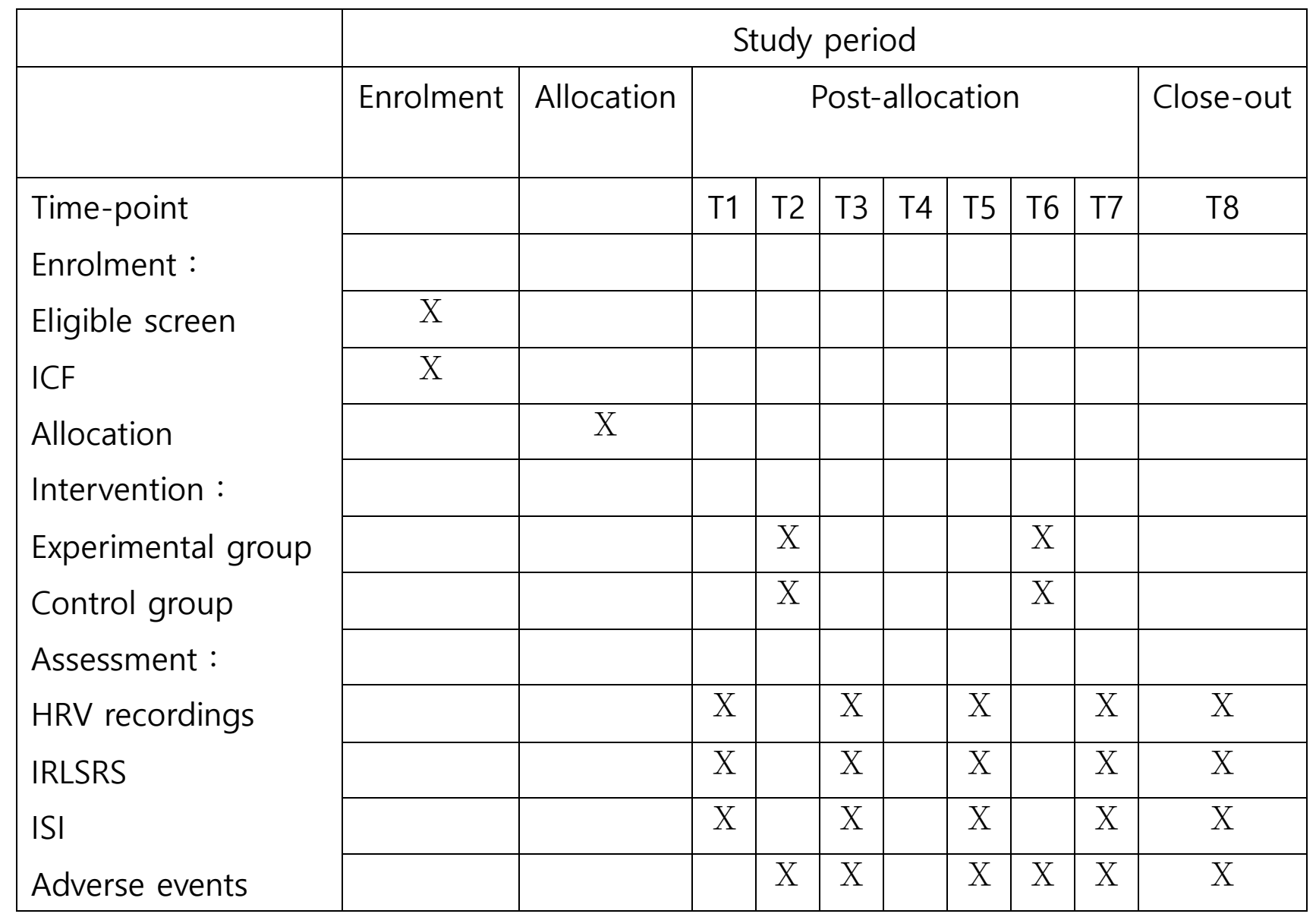

ICF : informed consent form

$\mathrm{T} 1$ : before $1^{\text {st }}$ intervention

T2 $: 1^{\text {st }}$ intervention, 8 treatments $/ 4$ weeks

T3 : after $1^{\text {st }}$ intervention

T4 : a 2-week washout period

T5 : before $2^{\text {nd }}$ intervention

T6 $: 2^{\text {nd }}$ intervention, 8 treatments $/ 4$ weeks

$\mathrm{T} 7:$ after $2^{\text {nd }}$ intervention

T8 : 1 month after the last treatment 


\section{Participant timeline $\{13\}$}

In table 2

\section{Sample size $\{14\}$}

This study is a pilot study for evaluating the efficacy of EA compared to sham EA, and to evaluate the feasibility of large clinical trials. In the hopes of decreasing the subject dropout rate, the trial is designed to only last 4 weeks. The minimum sample size for estimating the difference in the mean change in activity level of HRV between the experimental and control groups during the study period with an effect size of 0.54 (means of 3.3 and -3.7, standard deviations of 13.8 and 12.3 , respectively; $\alpha=0.05 ; 1-\beta=0.8$ ). Assuming a drop-out rate of $10 \%$, the desired sample size for this pilot study is 112 subjects, with 56 in each group.

Based on the a priori calculation in the statistical software G*Power 3.1.9.2, it was determined that a minimum total sample size of 124 would be needed in the independentsamples t-test to show a significant difference.

\section{Recruitment $\{15\}$}

Patients meeting the IRLSRS criteria will be recruited primarily through advertisements on hemodialysis room, outpatient clinics, and publicity at community service centers.

\section{Assignment of interventions: allocation}

\section{Sequence generation $\{16 a\}$}

This study is a randomized block designs by a computer-generated randomization list. Randomization allocation will be concealed from the physician, subjects and evaluators.

\section{Concealment mechanism $\{16 b\}$}

The group each subject is allocated to will be concealed in sequentially numbered sealed opaque envelopes.

\section{Implementation $\{16 c\}$}

The envelopes will only be opened after the subject has completed baseline clinical assessments. 


\section{Assignment of interventions: Blinding}

\section{Who will be blinded $\{17 \mathrm{a}\}$}

The trial participants, outcome assessors and data analysts are blinded in this study.

Due to the nature of acupuncture, masking of acupuncturists is quite difficult to achieve.

Patients, outcome assessors, and statisticians who perform the statistical analyses will be blinded to group assignment. Participant's allocated intervention will be not revealed until the statistical analysis is completed.

Procedure for unblinding if needed $\{17 b\}$

The same as $17 \mathrm{a}$

\section{Data collection and management}

\section{Plans for assessment and collection of outcomes $\{18 \mathrm{a}\}$}

All researchers including physicians, outcome assessors, and statisticians will receive training regarding data management. Data will be inputted into the medical record form of electronic computer which will be established before recruitment. The research assistant are responsible for collecting and verifying the accuracy of data.

\section{Plans to promote participant retention and complete follow-up \{18b\}}

The study collects only what is absolutely necessary to answer the research question. Alleviate the need for long-term follow-up by using proxy measures with earlier end points. Select questionnaires/procedures with lesser response time without compromising the result. We complete questionnaires via telephone or send them in the mail. We offer evening (after work hours) or weekend follow-up visits, and systematically organize trial procedures so that the patient moves quickly through the visits.

\section{Data management $\{19\}$}

The research assistant will keep tracking the direction (conditions) of post-treatment participants. Any personal information will remain confidential in a safe. Only the principal investigators can get the assessment. 


\section{Confidentiality $\{27\}$}

This study-related information will be stored securely at the study site. All participant information will be stored in locked file cabinets in areas with limited access. All laboratory specimens, reports, data collection, process, and administrative forms will be identified by a coded ID number only to maintain participant confidentiality. All records that contain names or other personal identifiers, such as locator forms and informed consent forms, will be stored separately from study records identified by code number.

All local databases will be secured with password-protected access systems. Forms, lists, logbooks, appointment books, and any other listings that link participant ID numbers to other identifying information will be stored in a separate, locked file in an area with limited access.

\section{Plans for collection, laboratory evaluation and storage of biological specimens for genetic or molecular analysis in this trial/future use $\{33\}$}

No samples collected

\section{Statistical methods}

\section{Statistical methods for primary and secondary outcomes $\{20 \mathrm{a}\}$}

Statistical analysis will be performed by using the software program Statistical Package for the Social Sciences (SPSS 22.0, IBM Corp., Armonk, NY). Participants' demographic information and levels of major measured variables were analyzed by descriptive statistics. The clinical indices will be calculated, after which the evaluation between the two groups will be compared using Student's t-test for continuous data and Chi-square or Fisher's exact test for categorical data. The difference of evaluation scores before and after each examination point will be analyzed using paired t-tests.

A mixed model will by used to assess the quality of the cross-over design, wherein the dependent variable is the clinical index and the main independent variables are acupuncture type, administration order, and their interaction. After testing the interaction, if there is no significant carry-over effect in the interaction model and no significant order effect in the main effect model, it could be confirmed that the quality of the cross-design in 
this study is acceptable.

The generalized estimating equation (GEE) to fit a linear regression model will be used to analyze longitudinal data of the two acupuncture modes for each clinical index across the 4 data collection points (treatments for 8 weeks) that yields robust standard errors estimated in the model. A value of $\mathrm{P} \& \mathrm{lt} ; 0.05$ will be regarded as statistically significant for the above statistical analyses.

\section{Interim analyses $\{21 b\}$}

An interim-analysis is performed on the primary endpoint when $50 \%$ of patients have been randomised and have completed the 4 weeks follow-up. The interim-analysis is performed by an independent statistician, blinded for the treatment allocation. The statistician will report to the independent DSMC [data and safety monitoring committee]. The DSMC will have unblinded access to all data and will discuss the results of the interim-analysis with the steering committee in a joint meeting. The steering committee decides on the continuation of the trial and will report to the central ethics committee.

\section{Methods for additional analyses (e.g. subgroup analyses) \{20b\}}

We plan to conduct two subgroup analyses, both with strong biological rationale and possible interaction effects. Subgroup analyses involve splitting all the participant data into subgroups, often so as to make comparisons between them. Subgroup analyses may be done for subsets of participants, or for subsets of studies.

\section{Methods in analysis to handle protocol non-adherence and any statistical methods to handle missing data $\{20 \mathrm{c}\}$}

We propose to test non-inferiority using two analysis sets; the intention-to-treat set, considering all patients as randomized regardless of whether they received the randomized treatment, and the "per protocol" analysis set.

\section{Plans to give access to the full protocol, participant level-data and statistical code} $\{31 \mathrm{c}\}$

We will deliver a completely de-identified data set to an appropriate data archive for sharing purposes. 


\section{Oversight and monitoring}

Composition of the coordinating centre and trial steering committee $\{5 \mathrm{~d}\}$

The IRB of the Changhua Christian Hospital will overseeing the trial.

Composition of the data monitoring committee, its role and reporting structure $\{21 \mathrm{a}\}$ An independent Data and Safety Monitoring Board (DSMB) will be established to review and interpret data generated from the study.

\section{Adverse event reporting and harms $\{22\}$}

Subjects will be asked to report any adverse events they experience. The evaluator will then interview the subjects to confirm the validity of the adverse event. If these events occur during treatment, the physician will immediately stop the procedure and treat the adverse event. The evaluator will then file a report detailing the seriousness of the event, event onset date, relationship between the event and the treatment, possible causes of the event other than the trial itself, and other relevant data. The report will then be sent to the ethics committee. The ethics committee will then decide whether or not to modify the study protocol or remove the subject from the trial.

\section{Frequency and plans for auditing trial conduct $\{23\}$}

The DSMB will review the progress of the trial every three months independently from investigators and the sponsor and decide on any premature closure of the study.

Plans for communicating important protocol amendments to relevant parties (e.g. trial participants, ethical committees) $\{25\}$

The principal investigator will apply to submit an application the IRB of the Changhua Christian Hospital for the trial modifications.

\section{Dissemination plans $\{31 \mathrm{a}\}$}

The authors have been informed of publication and gave their consent. 


\section{Discussion}

Current drug treatments, like dopaminergic drugs, have been widely long term used for RLS. While some previous have showed an initial improvement in symptoms, longer studies and clinical experience are reported that either treatment efficacy decreases with time and dopaminergic augmentation has been reported to be the major cause for treatment failure in RLS[22].

Several lines of evidence suggest that patients with RLS have autonomic system abnormalities. Symptoms related to the autonomic nervous system have been more frequently reported in patients with RLS[23]. Whereas Cikrikcioglu et al. reported that elevated sympathetic activity might be beneficial in relieving RLS symptoms[24], Yıldız et al. indicated that there is a relationship between RLS and increased sympathetic cardiac modulation[25]. It shall pay high attention to understand the mechanisms of autonomic nervous system functions in hemodialysis patients with RLS

Traditional Chinese medicine has been shown to may have beneficial effects in RLS, for example, acupuncture. However, to our knowledge those trials were either nonrandomized and had a limited number of subjects, or were subjective assessments. A washout period trial between treatment sessions was not used in previous randomized crossover studies of acupuncture on RLS.

Our study is the first investigation to analyse the change of HRV after EA in hemodialysis patients with RLS. We did this washout period to 2 weeks to further reduce potential carryover effects. The decision to use the SDNN, TP, LF, HF, LF/HF as the primary endpoint reflects the standard approach at the time the study was planned. In addition, an ANSWatch $\AA$ monitor provides an objective assessment of leg activity. The IRLSRS and ISI scores have often been used in evaluating RLS in previous studies[26, 27]; however, they are subjective assessments so we used them as secondary endpoints in this study.

We hypothesize that EA can help patients improve the abnormal leg activity in hemodialysis patients with RLS. If our hypothesis is correct, we may recommend subjects to receive EA treatment. In other words, EA appears to be an effective alternative in terms of cost and safety.

\section{Trial status}

IRB (protocol No. : Y_105_0300) was initially approved on August 23, 2019. NCT04356794 was registered on April 22, 2020. Recruitment began in May 2020. 
Expected date when recruitment will be completed is October 31, 2021.

\section{Abbreviations}

RLS : restless leg syndrome

HRV : heart rate variability

EA : electroacupuncture

IRLSRS : International Restless Legs Syndrome Rating Scale

ISI : Insomnia Severity Index

TCM : Traditional Chinese Medicine

DSMC : data and safety monitoring committee

\section{Declarations}

\section{Acknowledgements}

We appreciate the researchers, who have supported us with their helpful suggestions. This research was funded by the Changhua Christian Hospital, Taiwan

\section{Authors' contributions \{31b\}}

JMC contributed to conception, design of the study, and manuscript writing.

PFC, YCC and LCL participated in the study conception and the analysis of the data. All authors read and approved the final manuscript in the Authors' contributions section.

\section{Funding $\{4\}$}

This trial is funded by the Changhua Christian Hospital, Taiwan (program number No. 92419) under its clinical research hospital program. The funding source had no role in the study design, writing of the report, or the decision to submit the paper for publication.

\section{Availability of data and materials $\{29\}$}

The research assistant will keep tracking the direction (conditions) of post-treatment 
participants. Any personal information will remain confidential in a safe. Only the principal investigators can get the assessment.

\section{Ethics approval and consent to participate $\{24\}$}

The Changhua Christian Hospital Ethical Review Board (IRB No. : 170217). Written, informed consent to participate will be obtained from all participants.

\section{Consent for publication $\{32\}$}

Not Applicable.

\section{Competing interests $\{28\}$}

The authors declare that they have no competing interests with respect to the current trial.

\section{Authors' information (optional)}

Lun-Chien Lo is the corresponding author of this study.

\section{References}

1. Rizzo, V., et al., Impairment of sensory-motor integration in patients affected by RLS. J Neurol, 2010. 257(12): p. 1979-85.

2. Schlesinger, I., et al., Restless legs syndrome in stroke patients. Sleep Med, 2015. 16(8): p. 1006-10.

3. Allen, R.P. and C.J. Earley, Restless legs syndrome: a review of clinical and pathophysiologic features. J Clin Neurophysiol, 2001. 18(2): p. 128-47.

4. Siddiqui, S., et al., Risk factors for restless legs syndrome in dialysis patients. Nephron Clin Pract, 2005. 101(3): p. c155-60.

5. Mucsi, I., et al., Restless legs syndrome, insomnia and quality of life in patients on maintenance dialysis. Nephrol Dial Transplant, 2005. 20(3): p. 571-7.

6. Al-Jahdali, H.H., et al., Restless legs syndrome in patients on dialysis. Saudi J Kidney Dis Transpl, 2009. 20(3): p. 378-85.

7. Kim, J.M., et al., Restless legs syndrome in patients on hemodialysis: symptom severity and risk factors. J Clin Neurol, 2008. 4(4): p. 153-7. 
8. Merlino, G., et al., Sleep disorders in patients with end-stage renal disease undergoing dialysis therapy. Nephrol Dial Transplant, 2006. 21(1): p. 184-90.

9. La Manna, G., et al., Restless legs syndrome enhances cardiovascular risk and mortality in patients with end-stage kidney disease undergoing long-term haemodialysis treatment. Nephrol Dial Transplant, 2011. 26(6): p. 1976-83.

10. Billman, G.E., The effect of heart rate on the heart rate variability response to autonomic interventions. Front Physiol, 2013. 4: p. 222.

11. Izzi, F., et al., Is autonomic nervous system involved in restless legs syndrome during wakefulness? Sleep Med, 2014. 15(11): p. 1392-7.

12. Barone, D.A., et al., Heart rate variability in restless legs syndrome and periodic limb movements of Sleep. Sleep Sci, 2017. 10(2): p. 80-86.

13. Pan, W., et al., Actigraph evaluation of acupuncture for treating restless legs syndrome. Evid Based Complement Alternat Med, 2015. 2015: p. 343201.

14. Lee, J.H., et al., Comparison of electroacupuncture frequency-related effects on heart rate variability in healthy volunteers: a randomized clinical trial. J Acupunct Meridian Stud, 2011. 4(2): p. 107-15.

15. Grant, S., et al., CONSORT-SPI 2018 Explanation and Elaboration: guidance for reporting social and psychological intervention trials. Trials, 2018. 19(1): p. 406.

16. Heide, A.C., et al., Effects of transcutaneous spinal direct current stimulation in idiopathic restless legs patients. Brain Stimul, 2014. 7(5): p. 636-42.

17. Xu, X.M., et al., Complementary and alternative therapies for restless legs syndrome: An evidence-based systematic review. Sleep Med Rev, 2018. 38: p. 158-167.

18. Chen, Z.X., et al., Sham Electroacupuncture Methods in Randomized Controlled Trials. Sci Rep, 2017. 7: p. 40837.

19. Jubb, R.W., et al., A blinded randomised trial of acupuncture (manual and electroacupuncture) compared with a non-penetrating sham for the symptoms of osteoarthritis of the knee. Acupunct Med, 2008. 26(2): p. 69-78.

20. Walters, A.S., et al., Validation of the International Restless Legs Syndrome Study Group rating scale for restless legs syndrome. Sleep Med, 2003. 4(2): p. 121-32.

21. Bastien, C.H., A. Vallieres, and C.M. Morin, Validation of the Insomnia Severity Index as an outcome measure for insomnia research. Sleep Med, 2001. 2(4): p. 297-307.

22. Garcia-Borreguero, D., I. Cano-Pumarega, and R. Marulanda, Management of treatment failure in restless legs syndrome (Willis-Ekbom disease). Sleep Med Rev, 2018. 41: p. 5060. 
23. Shneyder, N., et al., Autonomic complaints in patients with restless legs syndrome. Sleep Med, 2013. 14(12): p. 1413-6.

24. Cikrikcioglu, M.A., et al., Oxidative stress and autonomic nervous system functions in restless legs syndrome. Eur J Clin Invest, 2011. 41(7): p. 734-42.

25. Yildiz, A., C. Yildiz, and A. Karakurt, Assessment of cardiac autonomic functions by heart rate variability in patients with restless leg syndrome. Turk Kardiyol Dern Ars, 2018. 46(3): p. 191-196.

26. Trenkwalder, C., et al., Treatment of restless legs syndrome: an evidence-based review and implications for clinical practice. Mov Disord, 2008. 23(16): p. 2267-302.

27. Carrier, J., et al., Effects of periodic leg movements during sleep in middle-aged subjects without sleep complaints. Mov Disord, 2005. 20(9): p. 1127-32. 


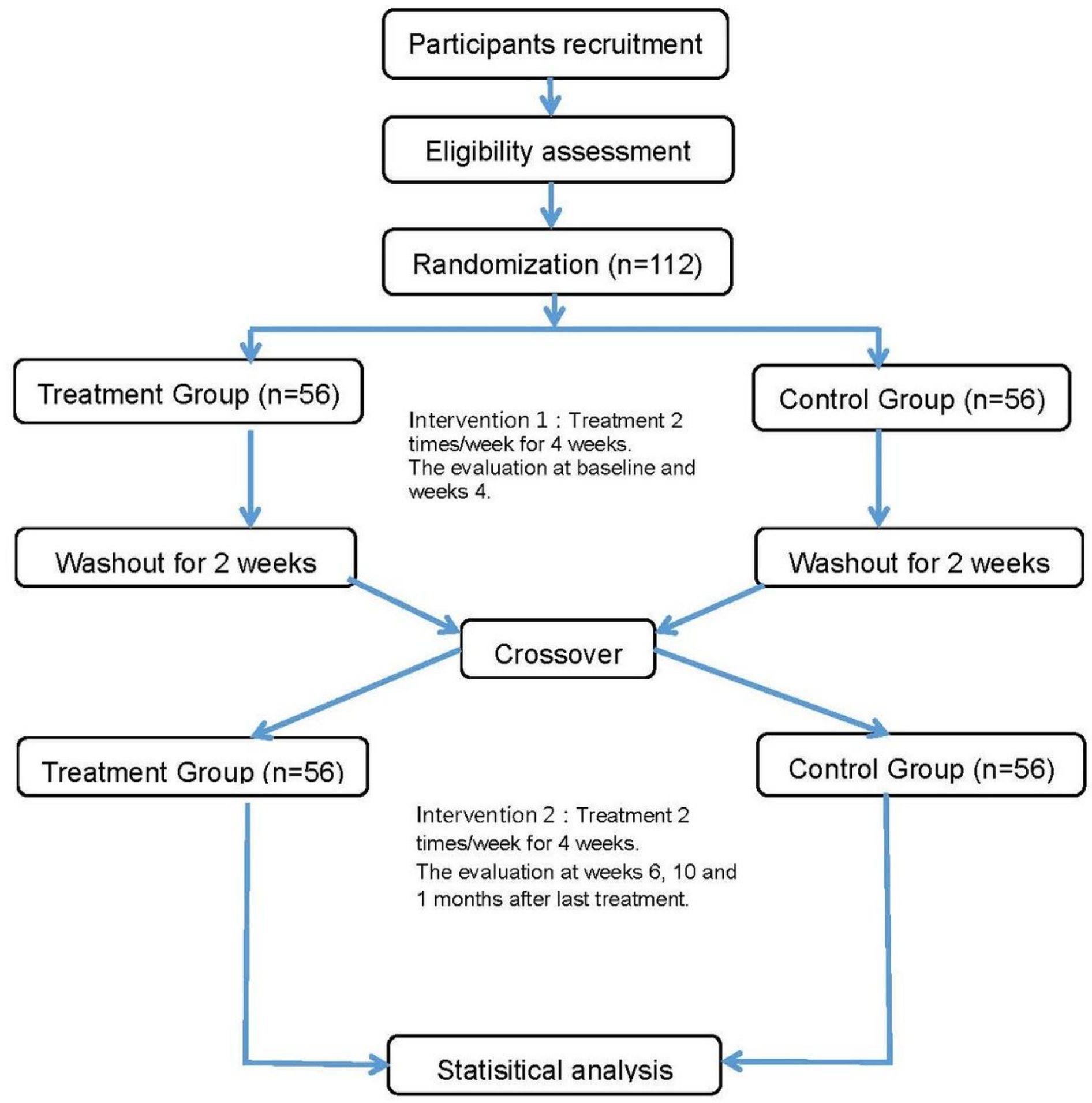

Figure 1

Flowchart of the study design 


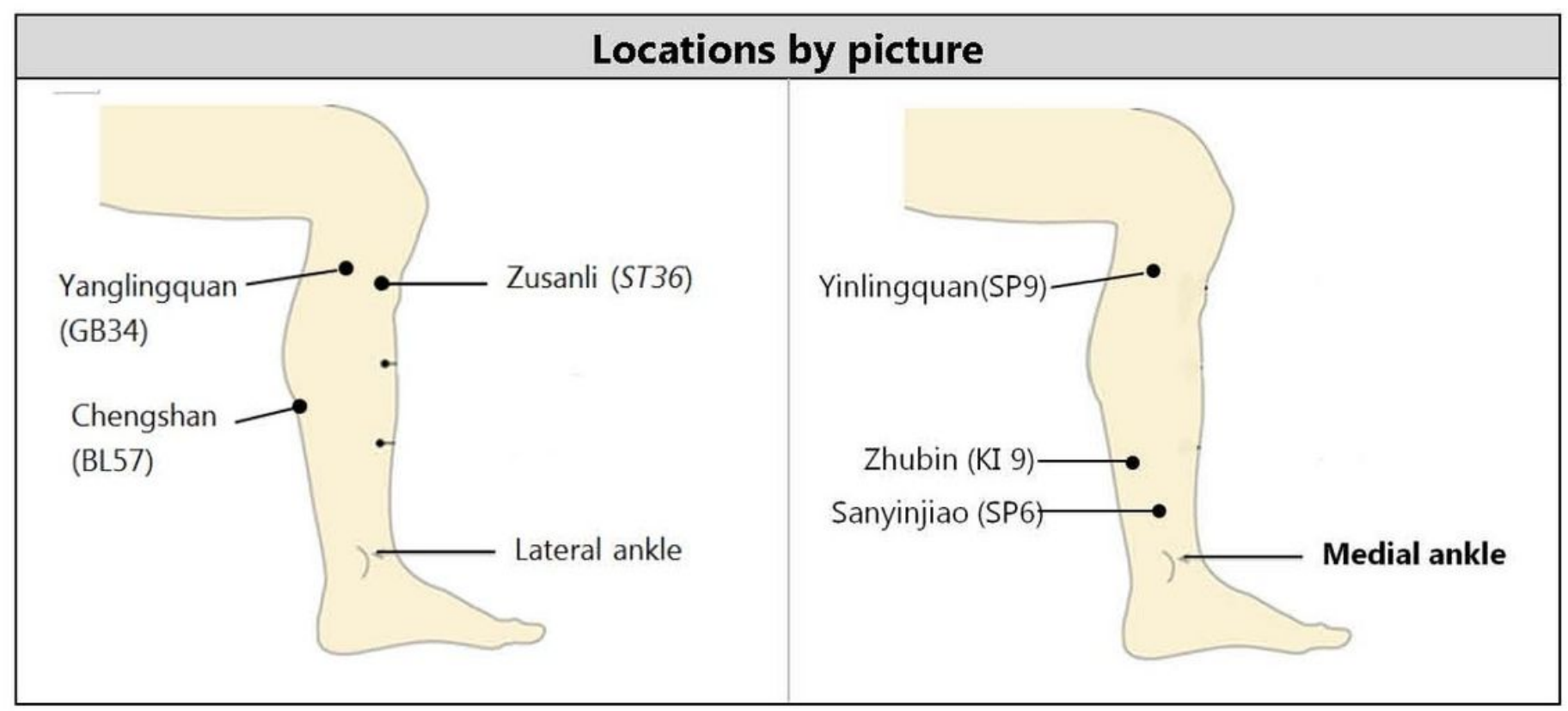

Figure 2

Locations of acupoints 